\title{
Promotion AND SuPPRESSION OF THERMAL Aggregation OF $\beta$-LACTOGLOBULIN BY ARgININE: A CONCENTRATION DEPENDENT MECHANISM
}

\author{
Jishnu Chakraborty $^{1}$ and Umesh Chandra Halder ${ }^{2}$ \\ ${ }^{1}$ Department of Chemistry, Camellia Institute of Engineering and Technology, \\ Budbud, Burdwan, West Bengal, India \\ jis_john1@yahoo.co.in \\ ${ }^{2}$ Protein Chemistry Laboratory, Department of Chemistry, Jadavpur University, \\ 188, Raja S.C. MullikRoad,Kolkata, West Bengal, India \\ uhalder2002@yahoo.com
}

\begin{abstract}
Bovine $\beta$-lactoglobulin ( $\beta$-lg), consisting of pronounced $\beta$-sheet content, have been chosen as a model protein which on prolonged thermal treatment forms large molecular aggregates similar to Alzheimer's type amyloid fibrils. The effects of L-arginine (free base) in thermal aggregation process of $\beta$-lg were monitored at varying concentrations. Concentration dependent opposite behaviour has been reported here for the first time where 0.2-0.3 $M$ concentration was optimized as an apparent critical concentration above which arginine acts as a suppressor and at below it behaves as a promoter of aggregation of $\beta$-lg. Solubility study and SDS-PAGE pattern followed by densitometric analysis shows this fact. Solution behaviour of arginine and its self assemblyformation were evidenced with the help of circular dichroism $(C D)$ studies. The delocalized pi-pi $(n \rightarrow n)$ type of interaction is proposed to foster the energy stabilization during the attainment of planarity of the molecules accompanied with the self-clustering of arginine molecules.
\end{abstract}

\section{KEYWORDS}

Protein, Aggregation, Arginine, Aggregation \& Suppression

\section{INTRODUCTION}

L-Arginine (Arg) is one of the most commonly used suppressors of aggregation of protein and hormones in vitro [1-3]. Its general acceptability as a suppressor of protein aggregates is well known, but the mechanism of its action in molecular detail remains elusive in spite of having a series of progress with Arg and its derivatives also [4]. The effect of Arg on the in vitro refolding of protein was discovered serendipitously by a group of authors [5]. Interestingly L-arginine and its derivatives were widely used in the industrial production process for plasminogen activator and continued to be marketed as bio-pharmaceuticals to this days also. Arginine exhibited the best results in preventing the formation of aggregates of lysozyme as a model protein under 
thermal stress and refolding pathway from previously denatured state in comparison to other 14 amino acids [1]. The preventive actions of Arg have been also reported by Shiraki and coworkers with other eight kinds of proteins. It was stated that the suppression effect was independent of size and/or iso-electric point of the proteins [1]. Nonetheless, one novel derivative, arginine ethyl ester significantly prevents thermal inactivation and aggregation of lysozyme whereas only Arg and guanidine $(\mathrm{Gdn} . \mathrm{HCl})$ was less effective in case of heat labile proteins [6]. Though $\mathrm{Gdn} . \mathrm{HCl}$ and urea are well established as aggregation suppressor that weaken the hydrophobic intermolecular interactions of proteins [7-8]. Arginine does not facilitate refolding, but suppresses aggregation, with only minor effect on protein stability [9], while the solubility of aggregate prone molecules is enhanced. Other additives like, proline, glycerol, glycine and ethylene glycol have also been reported which are not enough to eliminate the problems of protein aggregation and mis-folding [8]. Recently it was also reported that di-amines [10], and poly-amines typically spermine and spermidine, prevent heat-induced inactivation and aggregation of lysozyme [11-12].

Different mechanisms have been proposed towards the understanding of the preventive role of Arg in protein aggregation. The influence of co-solvent additives to the aqueous protein solution may in many cases be rationalized by the dichotomy of solubilizationvs stabilization or saltingout and salting-in. Solubilising agents often destabilize the native conformations in solution while stabilizing agents reduce protein solubility. The stability of the native state of dissolved proteins is generally not affected by the presence of L-arginine. Earlier it was reported that L-arginine has been found to destabilize RNaseA[13] and Cytochrome C [14] slightly. On the other hand, Reddy et al (2005) hardly found any destabilizing effect of L-arginine. $\mathrm{HCl}$ up to concentrations level of 1.0 $\mathrm{M}$ in the case of hen egg white Lysozyme [15]. Arginine ethyl ester and L- Arginine amide had been identified to destabilize the native state of protein in solution [16]. Moreover enzymatic activity and native structure of $\mathrm{H}$. Slinarum nucleoside diphosphate kinase were found to be preserved in solution containing up to $2 \mathrm{M}$ Arginine. $\mathrm{HCl}$ [17]. Arginine not only suppresses the aggregation reaction but also exerts an influence on the solubility of proteins in equilibrium. It was shown to increase the equilibrium solubility of most proteinogenic amino acids, except Lvaline and L-isolucine [18] and of aromatic molecules like, pyrene[19] and coumarine [20]. Ltyrosine and L-tryptophan experience the greatest increase in solubility of all naturally occurring amino acids, when L-arginine is present as a co-solvent. Based on the pronounced effects of arginine. $\mathrm{HCl}$ on the solubility of model compounds, specific interaction between the guanidino group of L-Arginine and aromatic amino acid residues have been proposed [17, 21]. The surface tension, linked to co-solvent properties, of aqueous solutions of L-arginine. $\mathrm{HCl}$ significantly increases with increasing concentration which is distinctly opposite in behaviour of $\mathrm{Gdn} . \mathrm{HCl}$ that indicates the protein stabilization and decreased protein solubility. Thus preferential binding and preferential hydration consist of direct interactions between protein mediated by co-solvent in solution remains enigmatic till date $[13,22]$.

In this work, we have selected bovine $\beta$-lactoglobulin ( $\beta$-lg), consisting of pronounced $\beta$-sheet content, as a model protein which on prolonged thermal treatment forms large molecular aggregates similar to Alzheimer's type amyloid fibrils. The effects of L-arginine (free base) in thermal aggregation process of $\beta-\lg$ were monitored at varying concentrations. Concentration dependent opposite behaviour has been reported here for the first time where 0.2-0.3 M concentration was optimized as an apparent critical concentration above which arginine acts as a suppressor and at below it behaves as a promoter of aggregation. Solubility study was indicative of this paradoxical behaviour of arginine with the appearance of heterogeneity in solutions during 
heating process. Both native and SDS-PAGE pattern and densitometric analysis evidenced the fact, detected preliminary on solubility studies of heat treated $\beta-\lg$ solutions.

Therefore our immediate interest was to study of the structural pattern of arginine in solution at different concentration by which the suppression behaviour could be explained. The previous knowledge was that arginine at higher concentration may form self assemblies in solution in which the $\beta-\lg$ molecules can be encapsulated/ segregated and be stabilized so that thermal exposure fails to form high molecular aggregates. Recently a group of authors have reported that molecular clusture of arginine in solutions display hydrophobic surface by the organization of its three methylene groups [23]. Thus hydrophobic surface inhibits protein-protein interaction by the masking effect. Nonetheless, salt bridged stabilization of charged zwitterionic arginine aggregates in gas phase has also been reported by another group[24]. Far UV-CD studies were of concentration dependent arginine solutions were sufficient enough to explain the self-cluster formation. Red shift in positive CD signals is due to facile $n \rightarrow \Pi^{*}$ electronic transition with accompanied ionic association and hydrogen bond formation in between inter-playing guanidine and carbonyl groups. Staking of Arginine molecules exhibit the clustering of hydrophobic methylene groups. Role of hydrogen bond or the hydrophobic interactions facilitating the $n \rightarrow \Pi^{*}$ energy transfer and $\Pi-\Pi$ interactions in cluster stabilization have been proposed in this context.

\section{MATERIALS AND METHODS:}

This section contains the information about the essential reagents synthesized and/or procured from the different available sources and from the suppliers.

\subsection{Materials}

Bovine $\beta-\lg$ used was isolated and purified from fresh cow milk following the procedure described earlier. The final product was lyophilized and stored at $4^{\circ} \mathrm{C}$ [25]. The purity of the protein was ascertained by SDS-PAGE, where a single band was observed. Concentration of $\beta-\mathrm{lg}$ in solution was determined spectrophotometrically $\left(\varepsilon_{280}=17,600 \mathrm{M}^{-1} \mathrm{~cm}^{-1}\right)$. AR grade sodium dihydrogen phosphate and disodium hydrogen phosphate were purchased from Sisco Research Laboratory, India. AR grade hydrochloric acid, ammonia, methanol, acetic acid, glycerol, urea, sodium acetate (anhydrous) and ammonium sulphate were obtained from Merck, India.Larginine, L-lysine, acryl amide, bis- acryl amide, N, N, N, N'-tetramethylethylenediamine (TEMED), ammonium persulphate, SDS, bromophenol blue, coomassie blue, Tris buffer, were from Sigma Chemical Co., U.S.A. All other chemicals were of the highest purity available.

\subsection{Methods:}

The methodology part contain the different scientific techniques opted for better understanding the mechanistic path of our objective and those were solubility measurements of protein solution in presence of varying arginine concentration. Native and SDS-PAGE of heat treated solutions were carried out to understand the aggregation profile and in presence of arginine and consequently densitometrin analysis was performed. Circular Dichroism studies were carried out particularly to understand the formation of higher ordered structure of choral molecules like arginine etc. 


\subsubsection{Solubility measurement of heat treated $\beta$-lg solutions}

Solubility of heat treated $\beta-\lg$ solutions was measured according to standard procedures, with a little modification [1]. Protein solutions of different concentrations $\left(1 \mathrm{mg} \mathrm{mL}{ }^{-1}, 2 \mathrm{mg} \mathrm{mL}^{-1}\right)$ containing arginine $(0-1.0 \mathrm{M})$ in phosphate buffer $(\mathrm{pH} 6.5)$ were incubated at $80{ }^{\circ} \mathrm{C}$ for 1 hour duration followed by cooling down to room temperature. The solutions were centrifuged at 15000 $\times \mathrm{g}$ for $15 \mathrm{~min}$ at $4{ }^{\circ} \mathrm{C}$. Then optical densities of the aliquots of supernatant solutions were measured at $280 \mathrm{~nm}$. Inverse of optical density data signifies for the extent of aggregate formation in percent $(\%)$ while optical density directly stands for residual soluble protein amount. The presence of different soluble oligomers could not be discriminated through optical density $\left(\mathrm{OD}_{280 \mathrm{~nm}}\right)$ measurements indeed. All the data points were an average of triplicate measurements.

\subsubsection{Native and SDS-PAGE study}

Native and SDS-PAGE studies were performed to characterize the monomer, dimmer, soluble oligomers and high molecular aggregates of $\beta$-lg. Aliquots $(10 \mu \mathrm{L})$ of heat treated $\beta$-lg solutions containing different concentrations of arginine were loaded in the wells of the native and SDS gel slab. The appearance of bands of protein aggregates were visible after staining with Coomassie brilliant blue R-250 and followed by destaining the gel with methanol and acetic acid solutions in the usual way. Both the native and SDS-PAGE were carried out to confirm the suppression efficiency of arginine was for both the non-covalent and co-valent (disulfide linked, S-S) aggregation of $\beta-\lg$. Quantitative analysis of the aggregates was done after densitometric studies (Quantity One software, equipped with Biorad gel documenting instrument, Universal hood II-S. N. 76S/04602, Italy) of the band appeared in SDS-PAGE.

\subsubsection{CirculalDichroism studies}

All the Far UV-CD experiments were carried out in a JASCO J-815 spectropolarimeter at $25^{\circ} \mathrm{C}$ using a cylindrical cell of path length $1 \mathrm{~mm}$. Solutions were made in $10 \mathrm{mM}$ sodium phosphate buffer, $\mathrm{pH}$ 6.5. Solution of L-arginine and L-lysine (in phosphate buffer, $\mathrm{pH}$ 6.5), of the concentration range $0.03 \mathrm{M}$ to $1.0 \mathrm{M}$, were used for far UV CD spectra. Typical instrumental parameters used were: path length $=1.0 \mathrm{~mm}$, sensitivity=50 mdeg (for far UV) and, time constant $2 \mathrm{~s}$, step resolution $=0.2 \mathrm{~nm} /$ data, scan speed $20 \mathrm{~nm} / \mathrm{min}$. Far UV-CD spectra were scanned within 200-250 $\mathrm{nm}$. Each spectrum was the average of three consecutive scans. The smooth spectral profiles were achieved using the polynomial curve-fitting program. The CD results were expressed as mean residual ellipticity (MRE) in degree $\mathrm{cm}^{2} \mathrm{dmol}^{-1}$, which can be defined as $\operatorname{MRE}(\theta)=\theta_{\text {obs }}($ mdeg $) / 10 \times \mathrm{n} \times 1 \times \mathrm{Cp}$

Where $\theta_{\mathrm{obs}}$ is the elipticities in millidegrees, $\mathrm{n}$ is the number of amino acid residues $(162), \mathrm{l}$ is the path length of the cell in $\mathrm{cm}$ and $\mathrm{Cp}$ is the mole fraction. Far UV and near UV CD spectra of $\beta-\mathrm{Lg}$ were recorded and the corresponding buffer spectrum was subtracted from each of them.

\section{RESULTS AND DISCUSSIONS}

This section contains the results obtained from the above studies which have been presented in graphical/ image form for better understanding the exact role of arginine. This part also contain a vivid discussion on the basis of the results obtained and for the first time we have proposed the 
delocalized pi-orbital interactions of self clustering arginine molecules actively participate in the aggregation-inhibition process.

\subsection{Solubility and aggregation studies}

Amount of soluble $\beta$-lg present in heat treated solutions containing different concentrations of arginine have been presented in terms of solubility percentage (\%) in Figure 1. The figure shows that there was a cut off point of arginine concentration at which the soluble protein concentrations were minimum due to formation of large molecular weight aggregates.
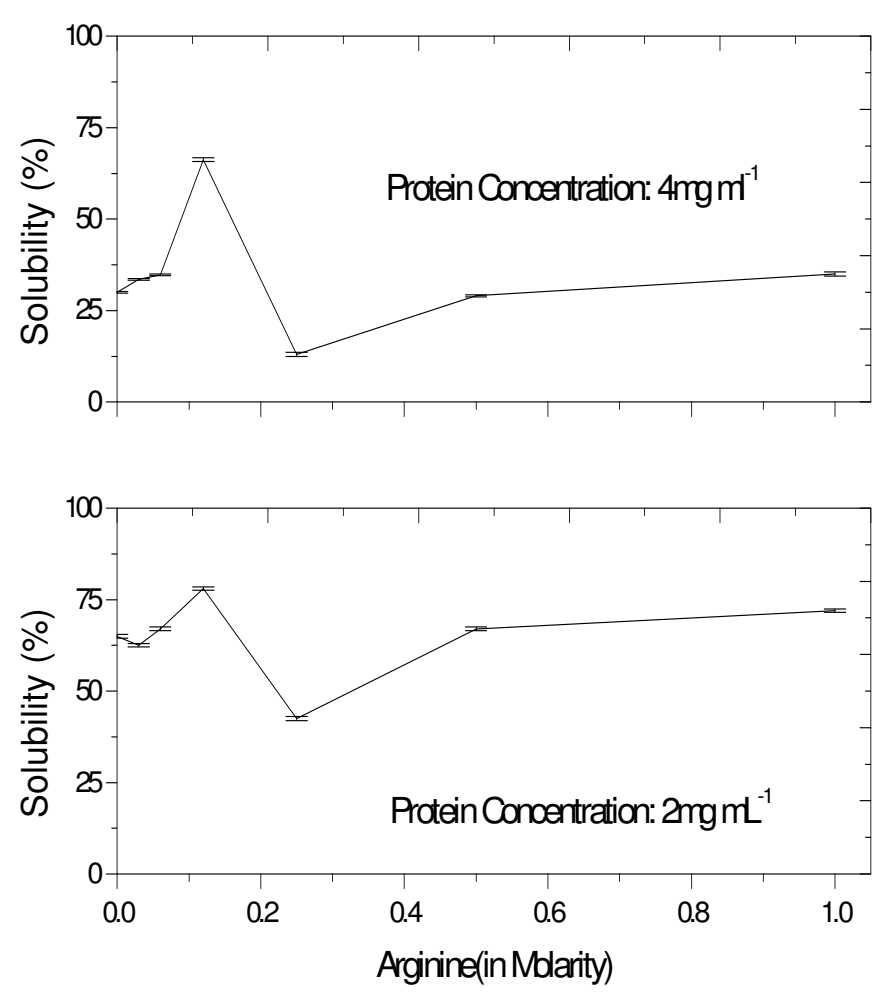

Figure 1: Solubility profile of $\beta$-lg (protein concentration: $4 \mathrm{mg} \mathrm{mL}^{-1}$ in upper panel and $2 \mathrm{mg} \mathrm{mL}^{-1}$ in lower panel) in presence of different arginine concentration in phosphate buffer (10 mM, pH 6.6-6.8).

It was necessary to mention that heterogeneities in solution were also observed with all the solutions of $\beta-\lg$ (both $2 \mathrm{mg} \mathrm{mL}^{-1}$ and $4 \mathrm{mg} \mathrm{mL}^{-1}$ ) containing arginine up to $0.25 \mathrm{M}$ concentration. Above this particular concentrations of arginine, the amount of soluble protein concentrations increases. This clearly indicates that mechanism of suppression of aggregation became effective after a certain concentration of arginine below which it acts as promoter of $\beta$-lg aggregation. This concentration of arginine was optimized 0.2-0.3 M. Arginine, in the concentration range of 0.5$1.0 \mathrm{M}$, almost suppressed the aggregation of $\beta-\lg$ where most of the $\beta-\lg$ remains in solutions. Similar trend was also observed in the case of suppression of lysozyme aggregation by arginine as reported elsewhere [1]. Appearance of heterogeneity/turbidity confirms the large aggregate formation, but solubility test could not insight about formation of soluble aggregates/ oligomers. 


\subsection{Native and SDS-PAGE studies: Densitometric analysis}

Characterization of aggregates was done by both native and SDS-PAGE techniques. Form the figure ( $2 \mathrm{a}$ and $2 \mathrm{~b}$ ), qualitatively it can be assumed that proportion of aggregates, particularly large molecular aggregates were formed at arginine concentration range 0.03 to 0.25 (lane $2,3,4,5$ ), where the aggregates failed to penetrate the gel (staking part) and band intensities in the monomeric region were sufficiently less with compared to dimer/ oligomeric bands. The band intensities in the monomeric region starts increasing with the gradual disappearance of the band at high molecular weight region ( staking gel part, after lane 4) with the gradual increase of arginine concentrations (lane 6, 7; Arginine concentration 0.5 amd 1.0 M respectively). Interestingly, the cause of appearance of heterogeneity during solubility studies was unfolded here and made some visible impact as the bands appeared in different molecular weight ranges. Nonetheless the contribution was not only because of the monomer but also the co-existence of oligomers in soluble forms was prominent even after heat treatment in all the lanes. Similar kind of observation of the existence of soluble oligomers was also reported [26]. Thus concentration dependent suppression of $\beta$-lg aggregation by arginine was nicely observed in gel-electrophoretic mobilities and SDS-PAGE also revealed that arginine was successful in suppression of covalently linked (di-sulfide linked, S-S) aggregation process where hydrophobic or electrostatic interactions could be discriminated.
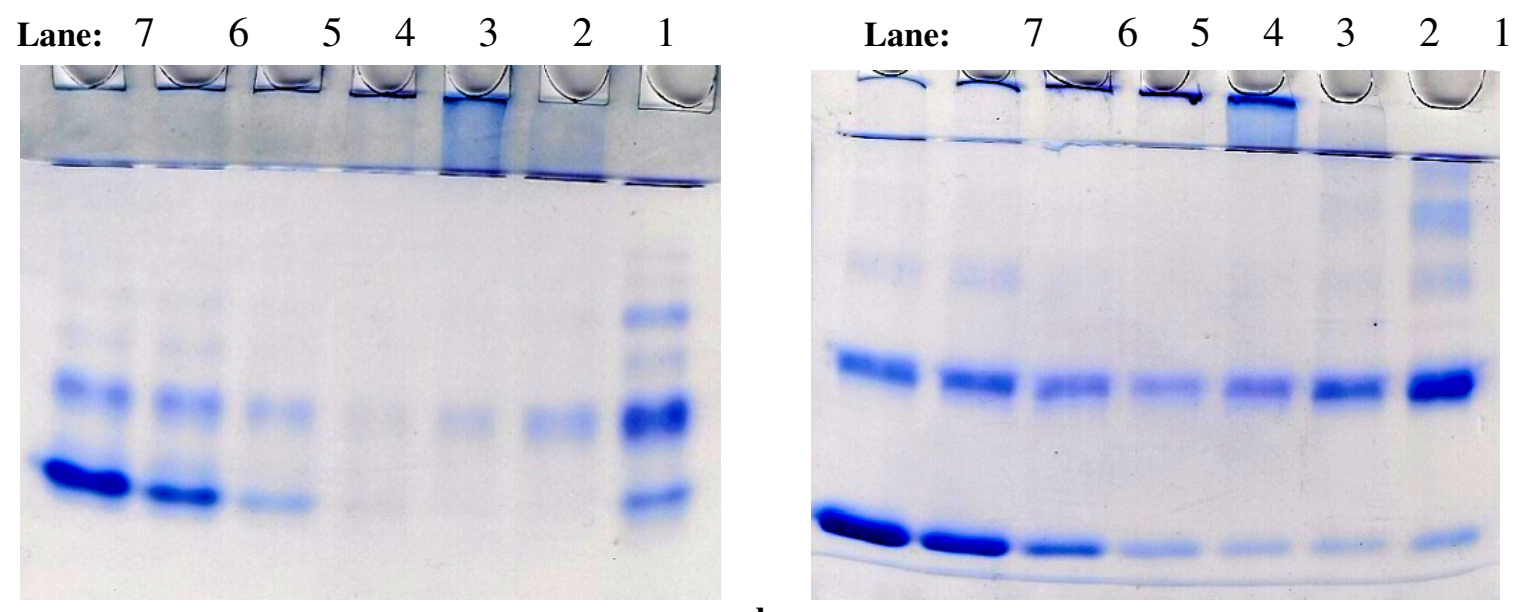

b

Figure 2: (a) Native PAGE and (b) SDS-PAGE pattern of heat treated $\beta$-lactoglobulin $\left(2 \mathrm{mg} \mathrm{mL}^{-1}\right)$ in presence of arginine (lane 1: $0 \mathrm{M}$, lane 2: $0.06 \mathrm{~m}$, lane 3: 0.12M, lane 4: $0.25 \mathrm{M}$, lane 6: $0.5 \mathrm{M}$, lane 7: $1 \mathrm{M}$ ) in Tris. $\mathrm{HCl}$ buffered solution ( $\mathrm{pH} 6.8)$.

Quantitative estimation of aggregate formation and arginine induced suppression was performed with the help of densitometric analysis of the bands appeared in the SDS-PAGE (Figure 3a). This estimation nicely demonstrates the monomer $\rightarrow$ oligomers/ aggregates $\rightarrow$ monomer transformation of $\beta-\mathrm{lg}$ depending on arginine concentrations. Partial loss of proteins due to heterogeneity appeared in heating process may be attributed toward the erroneous density profile (particularly in lane $3,4,5$ ) but gross errors within 10-20\% were in accountability. Lane wise densitometric intensity profile has been presented (Fig 3b) correspondingly. 

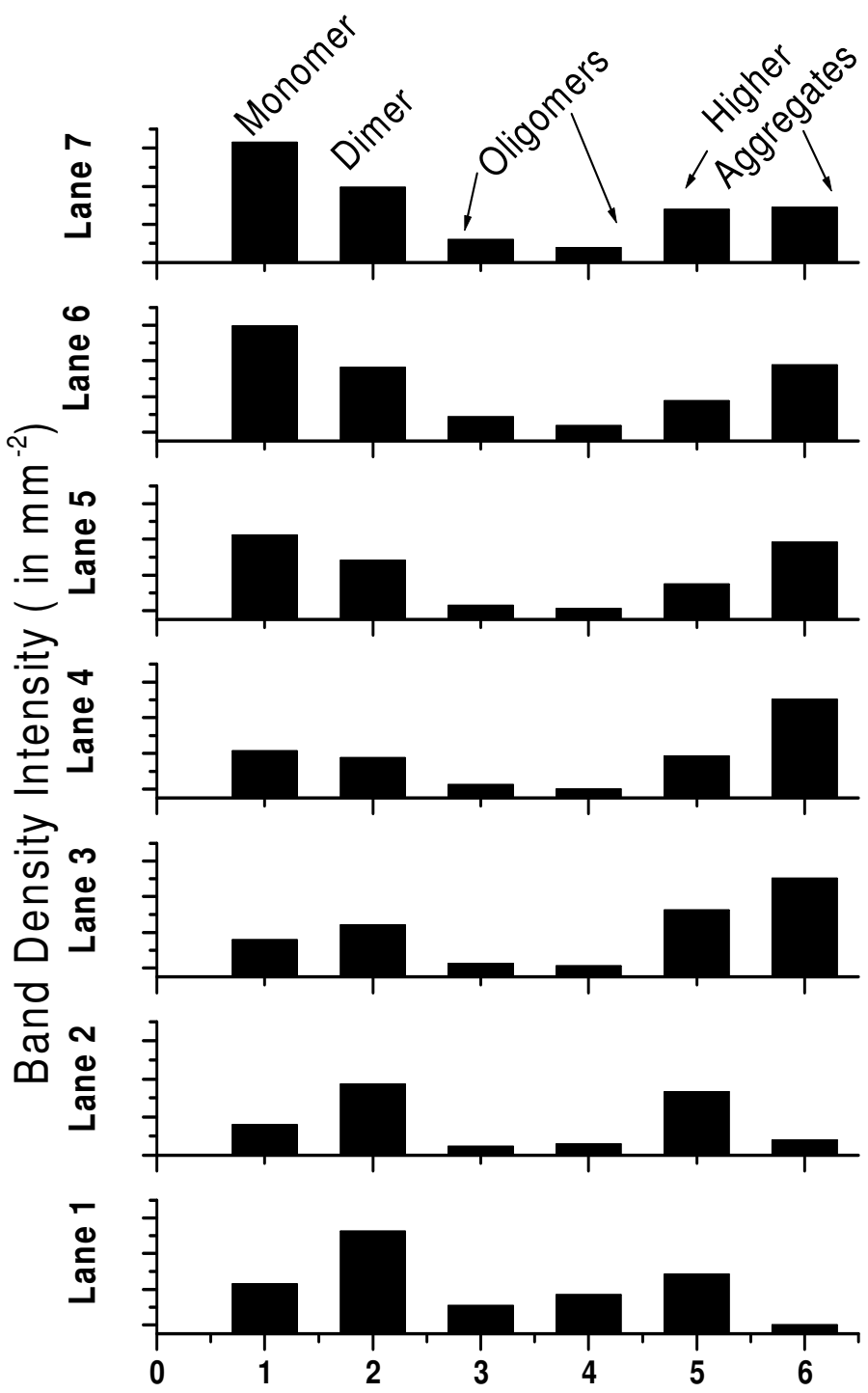

Figure 3a: Densitometric bar diagram of aggregation pattern and suppression in presence of Larginine from lane 1-7 in stacked fashion (Band density intensity $\mathrm{mm}^{-2}$ ). On X-axis, Monomer (1), Dimer (2), oligomers/ soluble aggregates (3-4) and high molecular weight aggregates (5-6) correspond to appearance of relative proportion of the bands after coomassie blue staining. Relative errors were within 10-20\%. 

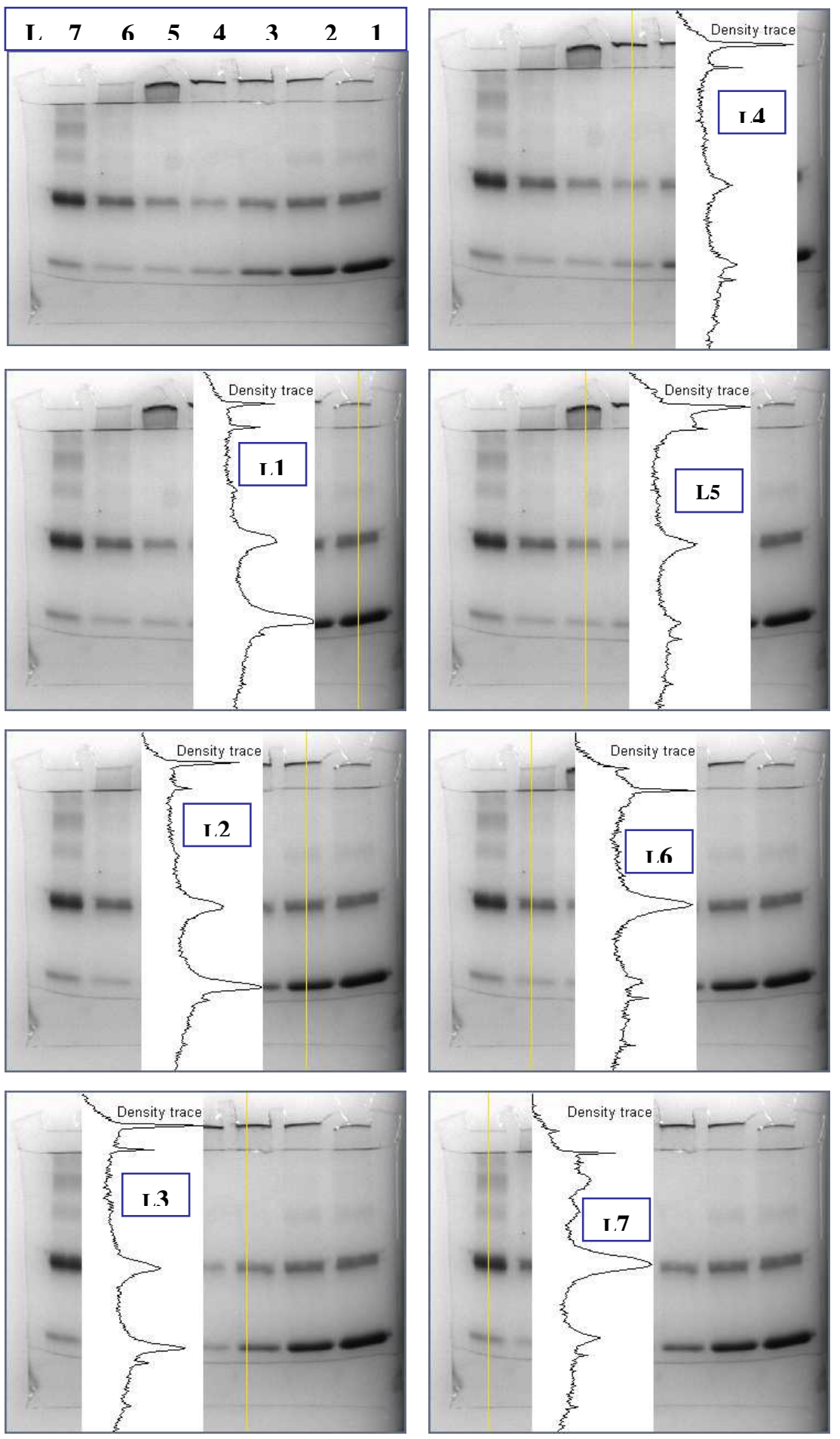

Figure 3b: Densitometry profile in SDS-PAGE pattern (top left) of $\beta$-lg aggregation promotion and suppression in presence of L-arginine from lane 1-7 (band density intensity $\mathrm{mm}^{-2}$ ). Density analysis was done with the help of software 'Qualtity One' equipped with the Biorad (Biorad, Universal Hood II-S.N. 76S/04602; Italy) gel documenting instrument. Relative errors were within 10-20\%. 


\subsection{Role of Arginine: a molecular level understanding}

It is evident that arginine is a novel suppressor in protein aggregation process at higher concentration. Some detail molecular level explanation is required to justify the statement. Several explanations have been proposed so far to support the mechanism. Earlier statement that hydrophilicity rather than hydrophobicity of the additives is the important factor for preventing aggregation was made by another group [1]. Okajono and co-workers have also proposed that high hydrophobicity is not a favourable characteristic of additives for suppression considering the hydrophobic chain length of polyamines [10]. It was also suggested that if side chain of arginine possesses properties identical to those of $\mathrm{Gdn} . \mathrm{HCl}$, incorrect inter- or intra-molecular hydrogen bonds may be cleaved and rearranged by arginine as in case by Gdn.HCl[27]. Even in our case it was quite inconclusive from the results of circular dichroism studies when secondary structural perturbation was monitored at identical $\mathrm{Gdn} . \mathrm{HCl}$ and arginine concentration along with $\beta-\lg$. At 1 $\mathrm{M}$ concentration $\mathrm{Gdn} . \mathrm{HCl}$ slightly perturbed $\beta-\mathrm{lg}$ secondary structural content where as at the same concentration, arginine signal was so prominent that fail to get any secondary conformational signature of $\beta-\lg$ (Figure 4). UV-CD studies were also performed at varying concentration of chirally pure L-lysine containing four hydrophobic methylene groups to verify the role of guanidino group in self-clustering of arginine molecules. At lower concentration of lysine, where guanidine part is not present, CD signal was obtained at $201 \mathrm{~nm}$ in buffered solution (pH 6.5) (Figure 5).

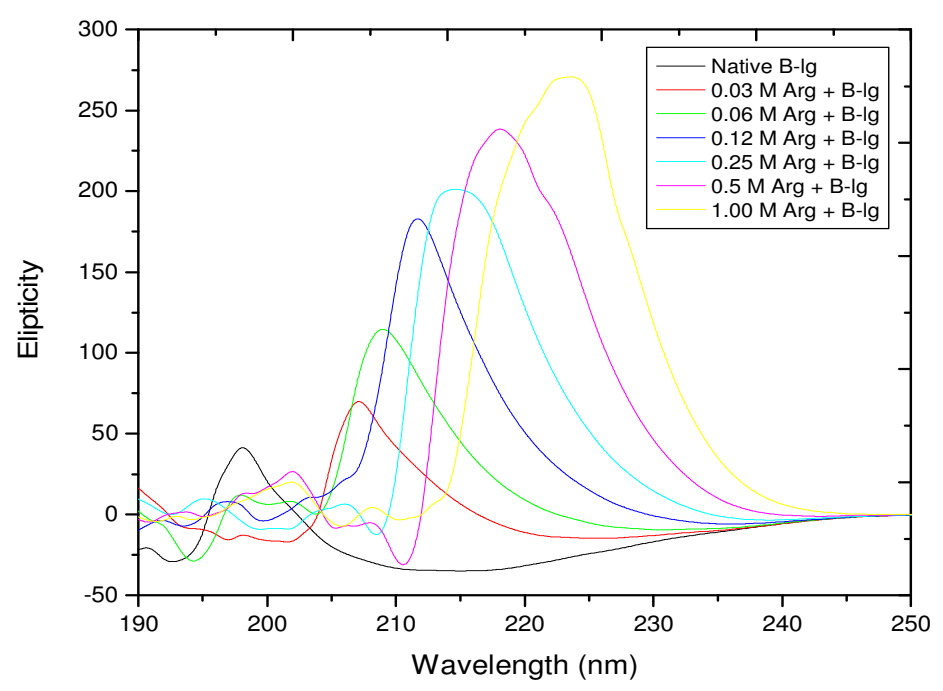

Figure 4: Far UV- CD spectral pattern of Native $\beta$-lg $\left(0.25 \mathrm{mg} \mathrm{mL}^{-1}\right.$, black line $)$ in presence of varying concentrations of arginine. Here the lines correspond as follows: Red, $0.03 \mathrm{M}$ Arginine+ $\beta-\mathrm{lg}$; green, 0.06 $\mathrm{M}$ Arg+ $\beta$-lg; blue, $0.12 \mathrm{M} \mathrm{Arg}+\beta-\mathrm{lg}$; cyan, $0.25 \mathrm{M} \mathrm{Arg}+\beta$-lg; pink, $0.5 \mathrm{M}$ Arg+ $\beta$-lg and yellow, $1.0 \mathrm{M}$ Arg $+\beta$-lg respectively. Solutions were prepared in phosphate buffer $(10 \mathrm{mM}, \mathrm{pH} 6.5)$ and spectra were recorded within the range of $200 \mathrm{~nm}$ to $250 \mathrm{~nm}$ at room temperature. All the data points were an average of three consecutive scans and correspondingly blank corrected.

This is probably because of the $n \rightarrow \Pi^{*}$ transition of carbonyl group present in L-lysine. In the case of $n \rightarrow \Pi^{*}$ transition of the carbonyl groups, substituted by an auxochrome, a large blue shift is observed from $280 \mathrm{~nm}$ to 200-220 nm (e.g. acetic acid: $204 \mathrm{~nm}$; acetamide: $220 \mathrm{~nm}$ ) [28]. With the increase in lysine concentrations (up to $1 \mathrm{M}$ ) a red shift $(212 \mathrm{~nm}$ ) in signals is found in UV-CD 
pattern, signifies $\Pi^{*}$ energy level stabilization. Generally amino acids have a general tendency to orient themselves in a peptide/ amide (-CONH-) like fashion with $\mathrm{N}$ - and C-terminal group juxtaposition rendering side chains away on the either sides [29]. Therefore the signals lie certainly in between $200-220 \mathrm{~nm}$, which is in good agreement with the literature values. At higher concentration, lysine form macromolecular aggregate/ micelle rather than an open ended bi-layer structures in aqueous solution [19]. Thus lowering of energy could be due to this macromolecular assembly of lysine residues. Therefore entire focus was directed towards the solution behavior of arginine through circular dichroism studies. Interestingly it was observed that signals, obtained from CD spectral patterns, of arginine put a signature of structure formation in aqueous solution (Figure 6). The sharp positive signal (at 207-209 nm) of arginine at lower concentration (0.03 M) was due to the $n \rightarrow \Pi^{*}$ electronic transition significantly contributed by the carbonyl moiety of carboxylate anion part, which ultimately shifted toward higher wavelength $(224 \mathrm{~nm})$ with the increase in arginine concentration (upto $1 \mathrm{M}$ ). This red shift (207 to $224 \mathrm{~nm}$ ) in CD signals was indicative of lowering of energy gaps accompanied with the energy stabilization up on increasing arginine concentration. The red shifts in $\mathrm{CD}$ signals have been recently mentioned in supramolecular/self-assembling peptide-amphiphilic systems [30-31].

This kind of lowering in energy may be due to self clustering of Arginine where the attainment of the different electronic microenvironment surrounding the carbonyl moiety can not be ignored. Here it is proposed that proximity of guanidino group and the carbonyl group of adjacent two Arginine molecules may foster an ionic association due to delocalization of $\Pi$-electrons where $\Pi-$ orbital interactions (pericyclic type, $4 \Pi+2 \Pi$ electronic system) could be possible (Scheme 1). Thus staking of Arginine molecules are made possible through weak $\Pi$-orbital interactions.

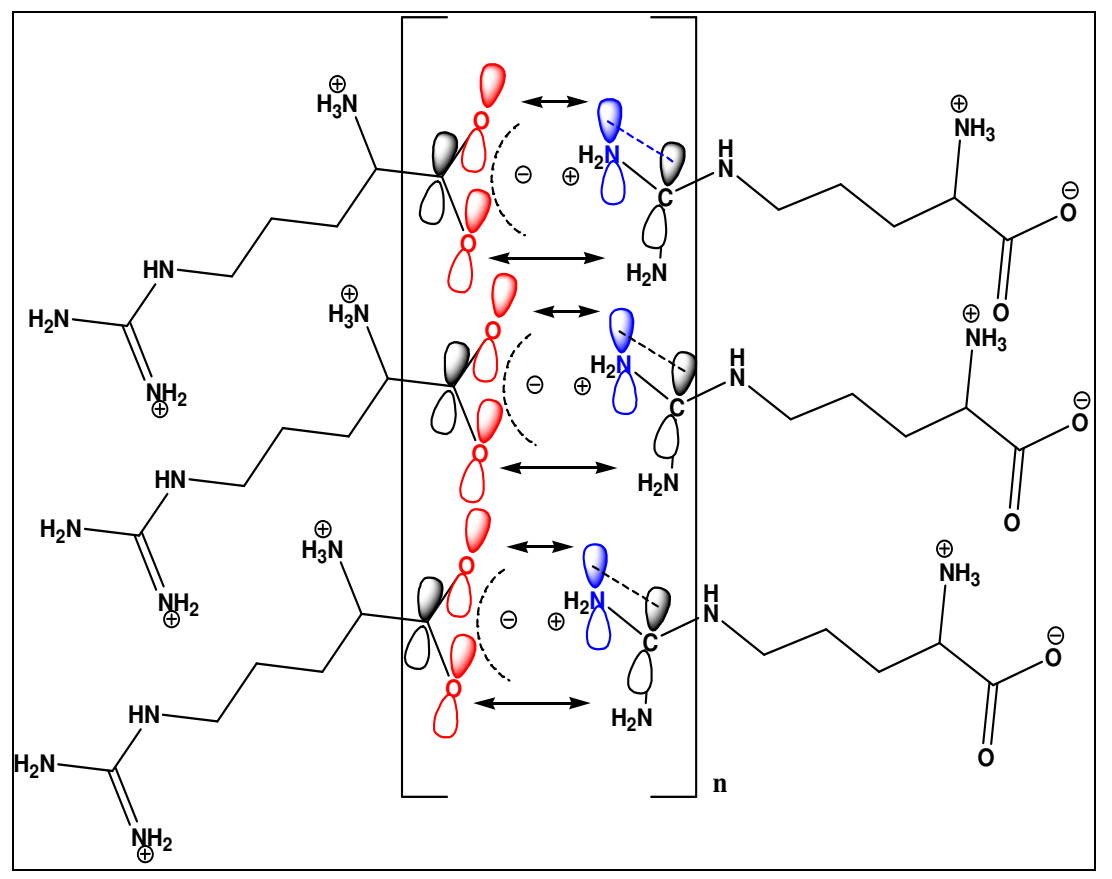

Scheme 1: Proposed schematic presentation of п-п interactions of arginine clusters. The delocalized 4п electrons from carboxylate groups and $2 \Pi$ electrons from guanidium groups of adjacent arginine molecules are expected to be stacked to provide extra stabilization in self-assembly. 
Unlike other amino acids, Arginine stacks in head-to tail fashion and a hydrophobic column composed of the three methylene groups is seen along one crystallographic axis [32]. Probably the interactions involved, the strong intermolecular interactions and other less significant secondary interactions during cluster formation was reported by a group of authors [24]. This kind of typical orientation and close packing have also been found in many crystal structures of arginine [33-34]. These cluster may have conformational properties as observed in crystal patterns and supposed to be rod shaped as seen in case of chirally pure praline molecule [35]. Still the role of intercalating water molecule might be highly significant in hydrogen bond formation of the clustering units in aqueous solution [36]. Das and co-workers also showed that molecular cluster of arginine in solution display a hydrophobic surface by the alignment of its three methylene groups, which ultimately interacts with protein and manifests a masking effect toward the protein molecules [23]. Therefore the characteristic organization of arginine leading to a columnar or rod like cluster may be stabilized by ionic association and/ or hydrogen bond formation and also by п-п type interactions with proximity of guanidino and/or carboxylate groups.

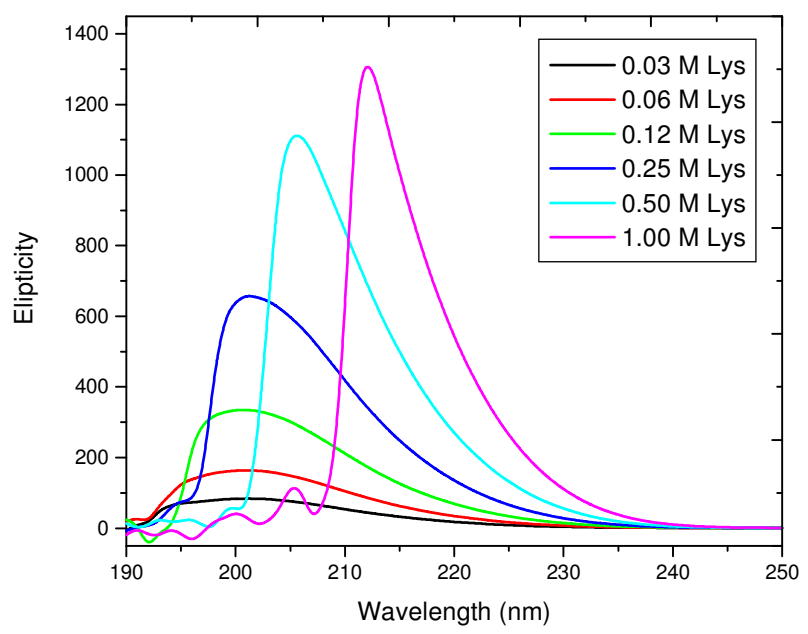

Figure 5: Far UV- CD spectral pattern of L-Lysine (Lys). Here the lines correspond as follows: black, 0.03 M Lys; red, 0.06 M Lys; green, 0.12 M Lys; blue, 0.25 M Lys; cyan, 0.5 M Lys and pink, 1.0 M Lys, respectively. Solutions were prepared in phosphate buffer $(\mathrm{pH} 6.5)$ and spectra were recorded within the range of $200 \mathrm{~nm}$ to $250 \mathrm{~nm}$ at room temperature. All the data points were an average of three consecutive scans and correspondingly blank corrected. 


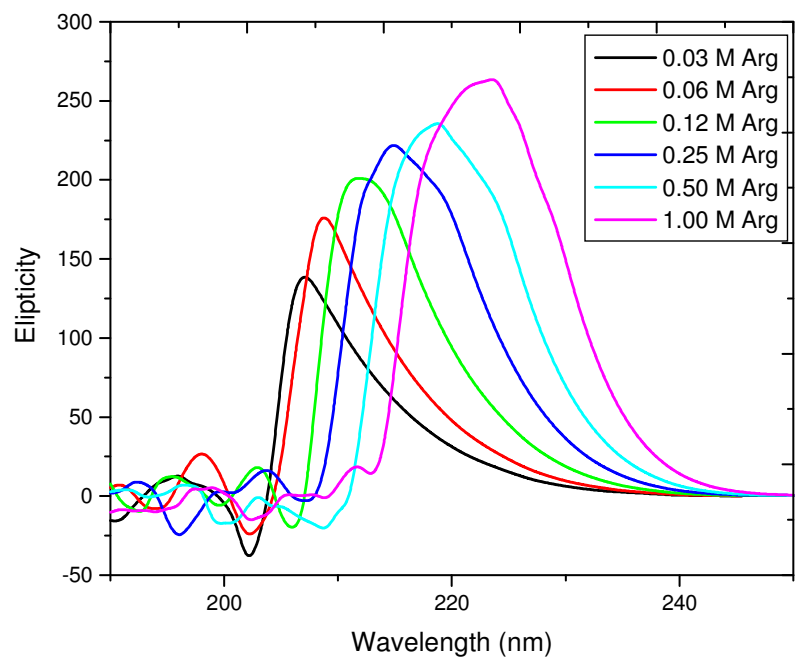

Figure 6: Far UV- CD spectral pattern of L-Arginine (Arg). Here the lines correspond as follows: black, $0.03 \mathrm{M} \mathrm{Arg}$; red, $0.06 \mathrm{M} \mathrm{Arg}$; green, $0.12 \mathrm{M} \mathrm{Arg}$; blue, $0.25 \mathrm{M} \mathrm{Arg}$; cyan, $0.5 \mathrm{M}$ Arg and pink, $1.0 \mathrm{M} \mathrm{Arg}$, respectively. Solutions were prepared in phosphate buffer $(\mathrm{pH} 6.5)$ and spectra were recorded within the range of $200 \mathrm{~nm}$ to $250 \mathrm{~nm}$ at room temperature. All the data points were an average of three consecutive scans and correspondingly blank corrected.

Nevertheless, it remains confusing whether hydrophobic association made this $n \rightarrow \Pi^{*}$ interaction favorable or the hydrogen bonding itself. On other side, the accumulation of delocalized $\Pi-$ orbitals in a stacked fashion makes the ground state energy levels electronically more populated that enhance the $\Pi \rightarrow \Pi^{*}$ transition easier, hence the red shift in CD signals occurs. Forces involved in the stacked planner cluster of Arginine have been demonstrated as strong multiple polar interactions elsewhere [23]. Indeed, the combination of these effects made the Arginine clustering possible. In other way these two effects are complementary to each other to form the columnar arginine cluster projecting a hydrophobic wall segregating and inhibiting the $\beta$-lg molecules prone to thermal aggregation. In the case of lysine, bathochromic shift in $n \rightarrow \Pi^{*}$ transition is mainly due to the hydrophobic interactions which stabilizes the micellerclusture. But in case of arginine, it is assumed that greater bathochromic shift compared to lysine is probably due to the $\Pi \rightarrow \Pi$ interactions $(4 \Pi+2 \Pi$ electrons) arising from the adjuscent carboxylate and guanodino group of two arginine residues. Water mediated hydrogen bonding and hydrophobic interactions contribute largely in both the cases.

\section{Conclusions}

In this study, it was observed that aggregation and suppression of $\beta-\lg$ is dependent upon concentration of arginine. Arginine fails to suppress the oligomers/ aggregates formation upto 0.25-0.3 M, above which its suppressive action becomes prominent. The cause of suppression of arginine at higher concentration is embedded in the arrangement of the self-clustering of arginine molecules. A pi-pi $(\Pi \rightarrow \Pi)$ type of interaction is proposed to foster the energy stabilization during the attainment of planarity of the molecules. This interaction helps in the projection of hydrophobic methylenic wall that helps to segregate $\beta$-lg molecules to segregate reducing protein-protein interactions. These experimental results help us to precede further to monitor the 
energy stabilization in factors within the scope of computational bio-physics and/or to explore the new horizon in some model based protein aggregation-suppression phenomena through computational-biology.

\section{ACKNOWLEDGEMENTS}

Financial support of UGC-CAS (Govt. of INDIA), Department of Chemistry, Jadavpur University, Kolkata is gratefully acknowledged. Authors also like to acknowledge Camellia Institute of Engineering \& technology, Budbud, Burdwan, WB, India, for providing academic and administrative help.

\section{REFERENCES}

[1] Shiraki, K., Kudou, M., Fujiwara, S., Imanaka, T. \& Takagi M. (2002) "Biophysical effect of amino acids on the prevention of protein aggregation", J. Biochem. (Tokyo), Vol 132, pp591-595.

[2] Bajorunait, E., Sereikaite, J. \&Bumelis, V. A. (2007) "L-Arginine suppresses aggregation of recombinant growth hormones in refolding process from E. Coli inclusion bodies", Protein J.,Vol 26, pp547-555.

[3] Christian, L. \& Rainer, R., (2009) "Suppression of protein aggregation by L-Arginine", Curr. Pharm. Biotechnol., Vol 10, pp408-414.

[4] Lange, C., Patil, G. \& Rudolph, R. (2005) "Ionic liquids as refolding additives: N'-alkyl and N'-(whydroxyalkyl) N-methylimidazolium chlorides”, Protein Sci.,Vol 14, pp2693-2701.

[5] Rudolph, R. \& Fischer, S. (1990) US Patent 4933434.

[6] Shiraki, K., Kudou, M., Nishikori, S., Kitagawa, H., Imanaka, T. \& Takagi, M. (2004) "Arginine ethylester prevents thermal inactivation and aggregation of lysozyme", Eur. J. Biochem.,Vol 271, pp3242-3247

[7] Buchner,J\& Rudolph, R. (1991) "Renaturation, purification and characterization of recombinant Fabfragments produced in Escherichia Coli", Biotechnology, Vol 9, pp157-162.

[8] Rudolph, R. \&Lilie, H. (1996) "In vitro folding of inclusion body proteins", FASEB J.,Vol 10, pp4956.

[9] Arakawa, T. \&Tsumoto, K. (2003) "The effects of arginine on refolding of aggregated proteins: not facilitate refolding, but suppress aggregation”, Biochem. Biophys. Res. Commun., Vol. 304, pp148152.

[10] Okanojo, M., Shraki, K., Kudou, M., Nishikori, S. \& Takagi, M. (2005) "Diamines prevent thermal aggregation and inactivation of Lysozyme", J. Biosci. Bioengg.,Vol 100, pp556-561.

[11] Kudou, M., Shiraki, K., Fujiwara, S., Imanaka, T. \& Takagi, M. (2003) "Prevention of thermal inactivation and aggregation of Lysozyme by polyamines", Eur. J. Biochem.,Vol 270, pp4547-4554.

[12] Shiraki, K., Kudou, M., Aso, Y. \& Takagi, M. (2003) "Approach for prevention of heat-induced protein aggregation by small molecular additives", Sci. Technol. Adv. Mat., Vol 4, pp55-59.

[13] Lin, T. Y. \&Timasheff, S. N. (1996) "On the role of surface tension in the stabilization of globular proteins", Protein Sci.,Vol 5, pp372-381.

[14] Taneja, S. \& Ahmad, F. (1994) "Increased thermal stability of proteins in the presence of amino acids", Biochem J.,Vol 303, pp147-153.

[15] Reddy, K. R. C., Lilie, H., Rudolph, R.\& Lange, C. (2005) "L-Arginine increases the solubility of unfolded species of hen egg white lysozyme", Protein Sci.,Vol 14, pp929-935.

[16] Hamada, H. \&Shiraki, K. (2007) "L-Argininamide improves the refolding more effectively than Larginine", J. Biotechnol.,Vol 130, pp153-160.

[17] Ishibashi, M., Tsumoto, K., Tokunaga, M., Ejima, D., Kita, Y. \& Arakawa, T. (2005) "Is arginine a protein-denaturant", Protein Expr. Purif.,Vol 42, pp1-6. 
[18] Arakawa, T., Ejima, D., Tsumoto, K., Obeyma N., Tanaka, Y., Kita, Y. \&Timasheff, S. N. (2007) "Suppression of protein interactions by arginine: A proposed mechanism of the arginine effects", Biophys. Chem., Vol. 127, pp1-8.

[19] Das, U., Hariprasad, G., Ethayathulla, A. S., Manral, P., Das, T. K., Pasha, S., Mann, A., Ganguli, M., Verma, A. K., Bhat, R., Chandryan, S. K., Ahmed, S., Sharma, S., Kaur, P., Singh, T. P. \&Srinivasan, A. (2007) "Inhibition of protein aggregation: Supramolecular assemblies of Arginine hold the key", pLoS ONE (2) e1176.

[20] Hirano, A., Arakawa, T. \&Shiraki, K. (2008) "Arginine increases the solubility of coumarine: Comparison with salting-in and salting-out additives", J. Biochem., Vol 144, pp363-369.

[21] Tsumoto, K., Umetsu, M., Kumagai, I., Ejima, D., Philo, J. S. \& Arakawa, T. (2004) "Role of arginine in protein refolding, solubilisation, and purification", Biotechnol. Prog.,Vol 20, pp13011308.

[22] Kita, Y., Arakawa, T., Lin, T. Y. \&Timasheff, S. N. (1994) "Contribution of the Surface Free Energy Perturbation to Protein-Solvent Interactions", Biochemistry,Vol 33, pp15178-15189.

[23] Das, U. \&Srinivasan, A. (2008) "Protein Aggregation-Suppression: Molecular Clusters of Arginine are responsible", FASEB J. 2008 22:1057.2 [Meeting Abstract]

[24] Julian, R. R., Hodyss, R. \& Beauchamp, J. L. (2001) "Cooperative salt bridge stabilization of gasphase zwitterions in neutral arginine cluster”, J. Am. Chem. Soc.,Vol 123, pp3577-3583.

[25] Chakraborty, J., Das, N., Das, K. P., Halder, U. C. (2009) "Loss of structural integrity and hydrophobic ligand binding capacity of acetylated and succinylated bovine $\beta$-lactoglobulin", International Dairy J.Vol 19, pp 43-49.

[26] Liu, Y. D., Li, J. J., Wang, F. W., Chen, J., Li, P. \& Su, Z. G., (2007) “A newly proposed mechanism for arginine-assisted protein refolding — not inhibiting soluble oligomers although promoting a correct structure", Protein Express Purif.,Vol 51, pp235-242.

[27] Trivedi, V. D., Raman, B., Rao, C. M. \& Ramakrishna, T. (1997) "Co-refolding denatured-reduced hen egg white lysozyme with acidic and basic proteins", FEBS Lett.,Vol 418, pp363-366.

[28] Kalsi, P. S. (2004) Spectroscopy of Organic Compounds, 6thEdn, New Age International Publishers, New Delhi, India, Chapter 2, pp 37.

[29] Suresh, C. G. \&Vijayan, M. (1983) "X-ray studies on crystalline complexes involving amino acids and peptides. Head-to-tail arrangement and a specific interaction in the crystal structure of L-arginine acetate", Int. J. Pep. Res.,Vol 21, pp223-226.

[30] Pashuck, E. T., Cui, H. \&Stupp, S. I. (2010) "Tuning supramolecular rigidity of peptide fibers through molecular structure", J. Am. Chem. Soc.,Vol 132, pp6041-6046.

[31] Rexeisen, E. L., Fan, W., Pangburn, T. O., Taribagil, R. R., Bates, F. S., Lodge, T. P., Tsapatsis, M. \&Kokkoli, E. (2010) "Self-assembling of fibronectin mimetic peptide-amphiphilenanofibers", Langmuir., Vol 26, pp1953-1959.

[32] Karle, J. \& Karle, I. L. (1964) "An application of the symbolic addition method to the structure of Largini dehydrate", Acts Cyst., Vol 17, pp835-841.

[33] Saenger, W. \& Wagner, K. G. (1972) "An X-ray study of Hydrogen Bonding in the Crystalline LArginine Phosphate monohydrate complex", ActaCrystallgr B.,Vol 28, pp2237-2244.

[34] Recacha, R., Verdeguer, N. \&Subirana, J. A. (1997) "Structure and supramolecular packing features of the dipeptide Arg-Val acetate" J. peptide Res.,Vol 50, pp388-392.

[35] Myung, S., Fioroni, M., Julian, R. R., Koeniger, S. L. \&Baik, M. H. (2006) "Chirally directed formation of nanometer-scale praline clusters", J. Am. Chem. Soc.,Vol 128, pp10833-10839.

[36] Jensen, J. H. \& Gordon, M. S., (1995) "On the number of water molecules necessary to stabilize the glycine zwitterion”, J. Am. Chem. Soc.Vol 117, pp8159-8170. 


\section{AUTHORS}

Dr. JishnuChakraborty, currently working as an Assistant Professor,Department of Chemistry, Camellia Institute of Engineering \& Technology, Budbud, Burdwan, WB, India, has been awarded his Ph, D. Degree in Science from Jadavpur University, Kolkata, in the year of 2011 under the Supervision of Dr. U. C. Halder. He has completed his Master Degree from C. S. J. M. Kanpur University, (formerly, Kanpur University) in the year of 2005 and he is an Honoursgraduatein Chemistry from University of Calcutta (2003).He has published several research articles in different journals of international repute. He was selected for the "UGC-sponsored Meritorious Fellowship in Science, 2007-2008" in the Department of Chemistry, Jadavpur University, Kolkata. Now a Day, he is holding the position of HOD, Chemistry in CIET, Budbud, Burdwan.His research interest is in protein chemistry, structural biology, nano-bio composite materials.

Dr. Umesh Chandra Halder, Assistant Professor,Department of Chemistry, Jadavpur University, Kolkata, West Bengal, India, has been awarded his Ph, D. Degree in Science from University of Calcutta, Kolkata, under the Supervision of Prof. N. K. Sinha, in Protein Chemistry Laboratory,Bose Institute, Kolkata. He has completed his Bachelors and Master Degree from University of Calcutta(Presidency College). He has published many research articles in different reputed journals of national and internationallevel.He has also run several scientific projects under DBT, CSIR, DSTetc. since the very beginning of his career in Jadavpur University. He is

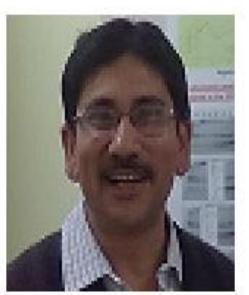

also a good scientific collaborator. His major research interest is in protein chemistry, bio-organic chemistry, biophysical chemistry and nano biology. 